Pacific Journal of Mathematic 


\title{
ON THE INVERSION FORMULA FOR THE CHARACTERISTIC FUNCTION
}

\author{
T. KAWATA
}

In the inversion formula

$$
F(x)-F(0)=\lim _{T \rightarrow \infty} \frac{1}{2 \pi} \int_{-T}^{T} \frac{e^{-i x t}-1}{-i t} f(t) d t
$$

for the characteristic function $f(t)$ of a distribution function $F(x)$, the limit of the symmetric integral is used. The purpose of this paper is to give a necessary and sufficient condition for the existence of the asymmetric improper integral $\lim _{T, T^{\prime} \rightarrow \infty} \int_{-T^{\prime}}^{T}$ on the right of the above formula.

Let $F(x)$ be a probability distribution function and $f(t)$ the corresponding characteristic function,

$$
f(t)=\int_{-\infty}^{\infty} e^{i t x} d F(x)
$$

We assume in this note that $F(x)$ is standardized so that

$$
F(x)=\frac{1}{2}[F(x+0)+F(x-0)] .
$$

The well known inversion formula states that

$$
F(x)-F(0)=\lim _{T \rightarrow \infty} \frac{1}{2 \pi} \int_{-T}^{T} \frac{e^{-i x t}-1}{-i t} f(t) d t
$$

for every $-\infty<x<\infty$.

It is also known that the symmetric integral of the right hand side cannot be replaces by the improper integral $\lim _{T, T^{\prime} \rightarrow \infty} \int_{-T^{\prime}}^{T^{\prime}}\left(T, T^{\prime}\right.$ going to infinity independently).

Actually we may easily see that

$$
\operatorname{Re}\left(\frac{1}{2 \pi} \int_{0}^{T} \frac{e^{-i x t}-1}{-i t} f(t) d t\right)=\operatorname{Re}\left(\frac{1}{2 \pi} \int_{-T}^{0} \frac{e^{-i x t}-1}{-i t} f(t) d t\right),
$$

and

$$
\operatorname{Im}\left(\frac{1}{2 \pi} \int_{0}^{T} \frac{e^{-i x t}-1}{-i t} f(t) d t\right)=-\operatorname{Im}\left(\frac{1}{2 \pi} \int_{-T}^{0} \frac{e^{-i x t}-1}{-i t} f(t) d t\right),
$$

and hence $\frac{1}{2 \pi} \int_{-T}^{T} \frac{e^{-i x t}-1}{-i t} f(t) d t$ cancels out its imaginary part.

The real part (1.4) always converges to $\frac{1}{2}[F(x)-F(0)]$. This gives 
the proof of (1.3). (See [1], pp. 263-264).

However the imaginary part (1.5) does not necessarily converge without some condition on $F(x)$. This is why the limit of the symmetric integral in (1.3) cannot be replaced by the general improper integral.

2. The condition for the existence of the improper integral. We shall give the necessary and sufficient condition for the existence of the limit of (1.5).

THEOREM 1. In order that the limit of (1.5) when $T \rightarrow \infty$ exists, it is necessary and sufficient that the integral

$$
\lim _{s \rightarrow 0+\infty} \int_{s}^{1} \frac{G(u, x)-G(u, 0)}{u} d u
$$

exists where,

$$
G(u, x)=F(u+x)-F(-u+x)
$$

and if (2.1) exists

$$
\lim _{r \rightarrow \infty} \operatorname{Im}\left(\frac{1}{2 \pi} \int_{0}^{T} \frac{e^{-i x t}-1}{-i t} f(t) d t\right)=\int_{0}^{\infty} \frac{G(u, \imath)-G(u, 0)}{u} d u .
$$

It must be noted that the integral of the right hand side of (2.3) exists in the neighborhood of the infinity. In fact $G(u, x)-G(u, 0)=$ $[F(u+x)-F(u)]-[F(-u+x)-F(-u)]$ and $F(u+x)-F(u) \in L$, $(-\infty, \infty)$ for every fixed $x$.

We shall now prove the theorem.

Let

$$
I(x, T)=\operatorname{Im}\left(\frac{1}{2 \pi} \int_{0}^{T} \frac{e^{-i x t}-1}{-i t} f(t) d t\right) .
$$

We then easily see that

$$
\begin{aligned}
I(x, T) & =\frac{1}{2 \pi} \int_{-\infty}^{\infty} d F(u) \int_{0}^{T} \frac{\sin x t \sin u t-(1-\cos x t) \cos u t}{t} d t \\
& =\frac{1}{2 \pi} \int_{-\infty}^{\infty} d F(u) \int_{0}^{T} \frac{\cos (u-x) t-\cos u t}{t} d t \\
& =\frac{1}{2 \pi} \int_{-\infty}^{\infty} d F(u) \int_{0}^{T} d t \int_{u-x}^{u} \sin v t d v \\
& =\frac{1}{2 \pi} \int_{-\infty}^{\infty} d F(u) \int_{u-x}^{u} \frac{1-\cos v T}{v} d v
\end{aligned}
$$




$$
\begin{aligned}
& =\frac{1}{2 \pi} \int_{-\infty}^{\infty} \frac{1-\cos v T}{v} d v \int_{v}^{v+x} d F(u) \\
& =\frac{1}{2 \pi} \int_{0}^{\infty}[G(v, x)-G(v, 0)] \frac{1-\cos v T}{v} d v .
\end{aligned}
$$

As was mentioned before, $G(v, x)-G(v, 0) \in L_{1}(-\infty, \infty)$. Hence the Riemann-Lebesgue lemma shows that

$$
\lim _{T \rightarrow \infty} \int_{\varepsilon}^{\infty}[G(v, x)-G(v, 0)] \frac{\cos v T}{v} d v=0
$$

for any $\varepsilon>0$. Therefore we may write

$$
\begin{aligned}
I(x, T)= & \int_{0}^{s} \frac{G(v, x)-G(v, 0)}{v}(1-\cos v T) d v \\
& +\int_{s}^{\infty} \frac{G(v, x)-G(v, 0)}{v} d v+o(1)
\end{aligned}
$$

as $T \rightarrow \infty$, for a fixed $\varepsilon>0$.

Now we shall show the sufficiency of the condition of the theorem. Let $\varepsilon>0$ be arbitrary but fixed. Write

$$
\begin{aligned}
& \int_{0}^{\varepsilon} \frac{G(v, x)-G(v, 0)}{v}(1-\cos v T) d v \\
= & \int_{0}^{1 / T}+\int_{1 / T}^{\varepsilon}=K_{1}+K_{2},
\end{aligned}
$$

say. We have

$$
\begin{aligned}
\left|K_{1}\right| & \leqq \int_{0}^{1 / T}|G(v, x)-G(v, 0)| \frac{1-\cos v t}{v} d v \\
& \leqq C T \int_{0}^{1 / T}|G(v, x)-G(v, 0)| d v,
\end{aligned}
$$

for some constant $C$.

$\lim _{v \rightarrow 0+}[G(v, x)-G(v, 0)]$ exists since $F$ is nondecreasing and it must be zero, otherwise (2.1) does not exist. Hence the last expression converges to zero.

$$
K_{1}=o(1), \quad \text { as } \quad T \rightarrow \infty
$$

Next write

$$
\chi(v)=G(v, x)-G(v, 0) .
$$

Choose $\varepsilon$ such that $|\chi(v)|<\delta$ for $|v| \leqq \varepsilon$ for an arbitrary chosen $\delta$. Since $\chi(v) / v$ is of bounded variation in $[1 / T, \varepsilon]$, we have, using the second mean value theorem, 


$$
\begin{aligned}
K_{2} & =\int_{1 / T}^{\varepsilon} \frac{\chi(v)}{v} d v-\int_{1 / T}^{\varepsilon} \frac{\chi(v)}{v} \cos v T d v \\
& =\int_{1 / T}^{\varepsilon} \frac{\chi(v)}{v} d v-T \chi\left(\frac{1}{T}\right) \int_{1 / T}^{\xi} \cos v T d v-\frac{\chi(\varepsilon)}{\varepsilon} \int_{\xi}^{\varepsilon} \cos v T d v
\end{aligned}
$$

for some $1 / T<\xi<\varepsilon$. Thus

$$
\left|K_{2}-\int_{1 / T}^{\varepsilon} \frac{\chi(v)}{v} d v\right| \leqq 2 \chi\left(\frac{1}{T}\right)+2 \chi(\varepsilon) \leqq 4 \delta .
$$

Therefore from (2.4) and (2.5)

$$
\left|I(x, T)-\frac{1}{2 \pi} \int_{1 / T}^{\infty} \frac{\chi(v)}{v} d v\right| \leqq \frac{2 \delta}{\pi}+o(1) .
$$

This shows the sufficiency of the condition of the theorem and gives (2.3).

We shall next show the necessity. Define $\chi(v)$ as before. We see that $\chi(v)$ has the limit $c$ as $v \rightarrow+0$. If $c \neq 0$, then from (2.4)

$$
\begin{aligned}
& I(x, T)-c \int_{0}^{\varepsilon} \frac{1-\cos v T}{v} d v \\
= & \int_{0}^{\varepsilon} \frac{[\chi(v)-c](1-\cos v T)}{v} d v+\int_{\varepsilon}^{\infty} \frac{\chi(v)}{v} d v+o(1) .
\end{aligned}
$$

The first integral of the right hand side is handled in the same way as in deriving (2.6) and (2.9) with $\chi(v)-c$ in place of

$$
\chi(v)=G(v, x)-G(v, 0) .
$$

Actually instead of (2.6) we see that $K_{1}$ with $\phi(v)-c$ is bounded by $C T \int_{0}^{1 / T}|\chi(v)-c| d v$ which is $o(1)$. In place of (2.9) we have

$$
\begin{aligned}
\mid I(x, T) & -\frac{c}{2 \pi} \int_{0}^{\varepsilon} \frac{1-\cos v T}{v} d v-\frac{1}{2 \pi} \int_{1 / T}^{\varepsilon} \frac{\chi(v)-c}{v} d v \\
& -\frac{1}{2 \pi} \int_{\varepsilon}^{\infty} \frac{\chi(v)}{v} d v \mid \leqq C_{1} \delta+o(1)
\end{aligned}
$$

where $C_{1}$ is some constant.

$$
\int_{0}^{\varepsilon} \frac{1-\cos v T}{v} d v=2 \int_{0}^{\varepsilon T} \frac{\sin ^{2} v / 2}{v} d v \geqq C_{2} \log \varepsilon T,
$$

where $C_{2}$ is an absolute constant. Choose $\varepsilon$ for an arbitrary given $\eta<C_{2}$ so that $|\chi(v)-c|<\eta$ for $0<v<\varepsilon$. Then

$$
\left|\int_{1 / T}^{\varepsilon} \frac{\chi(v)-c}{v} d v\right| \leqq \eta \log \varepsilon T .
$$


Hence if $I(x, T)$ has a limit as $T \rightarrow \infty$, then in view of (2.11) and (2.12), (2.10) implies a contradiction. Hence we have that $c=0$. Using (2.10), this yields

$$
\left|I(x, T)-\frac{1}{2 \pi} \int_{1 / T}^{\infty} \frac{\chi(v)}{v} d v\right| \leqq C_{1} \delta+o(1) .
$$

This proves the necessity of the condition.

3. Remarks. From Theorem 1, we immediately obtain

THEOREM 2. In order that

$$
\lim _{T, T^{\prime} \rightarrow \infty} \frac{1}{2 \pi} \int_{-T^{\prime}}^{T^{\prime}} \frac{e^{-i x t}-1}{-i t} f(t) d t
$$

exists, it is necessary and sufficient that (2.1) exists for $\varepsilon>0$. (The limit is $F(x)-F(0))$.

Similar arguments apply to the integral

$$
J_{1}(x, T)=\int_{1}^{T} \frac{f(t) e^{-i x t}}{i t} d t \text { and } J_{2}(x, T)=\int_{-T}^{-1} \frac{f(t) e^{-i x t}}{i t} d t .
$$

We easily see that $J_{1}$ and $J_{2}$ are conjugate complex. We may show that in order for $J_{1}(x, T)$ or $J_{2}(x, T)$ to converge as $T \rightarrow \infty$, it is necessary and sufficient that

$$
\int_{0}^{s} \frac{F(u+x)-F(-u+x)}{u} d u<\infty
$$

for some $\varepsilon>0$.

(3.1) implies that

$$
\lim _{T \rightarrow \infty} \frac{1}{2 \pi} \int_{-T}^{T} f(t) e^{-i x t} d t=0
$$

which is very well known when $F(x)$ is continuous at $x$. (3.1) says more than this about the improper integrability of $f(t)$ near infinity with the additional condition (3.2) on $F(x)$.

The sufficiency of (3.2) for the existence of the limits of (3.1) was proved in [2] before.

\section{REFERENCES}

1. T. Kawata, The characteristic function of a probability distribution, Tohoku Math. J. 48 (1941).

2. A. Rényi, Wahrscheinlichkeitsrechnung, Berlin, 1962.

Received November 20, 1968. This work was supported under National Science Foundation Grants GP-1577 and GP-3725. 



\section{PACIFIC JOURNAL OF MATHEMATICS}

\section{EDITORS}

H. ROYDEN

Stanford University

Stanford, California

Richard Pierce

University of Washington

Seattle, Washington 98105
J. DUGUNDJI

Department of Mathematics

University of Southern California

Los Angeles, California 90007

BASIL GORDON

University of California

Los Angeles, California 90024

\section{ASSOCIATE EDITORS}
E. F. BECKENBACH
B. H. NEUMANN
F. WOLF
K. YosHIDA

\section{SUPPORTING INSTITUTIONS}

UNIVERSITY OF BRITISH COLUMBIA

CALIFORNIA INSTITUTE OF TECHNOLOGY

UNIVERSITY OF CALIFORNIA

MONTANA STATE UNIVERSITY

UNIVERSITY OF NEVADA

NEW MEXICO STATE UNIVERSITY

OREGON STATE UNIVERSITY

UNIVERSITY OF OREGON

OSAKA UNIVERSITY

UNIVERSITY OF SOUTHERN CALIFORNIA
STANFORD UNIVERSITY

UNIVERSITY OF TOKYO

UNIVERSITY OF UTAH

WASHINGTON STATE UNIVERSITY

UNIVERSITY OF WASHINGTON

*

AMERICAN MATHEMATICAL SOCIETY CHEVRON RESEARCH CORPORATION TRW SYSTEMS

NAVAL WEAPONS CENTER 


\section{Pacific Journal of Mathematics}

\section{Vol. 31, No. $1 \quad$ November, 1969}

James Burton Ax, Injective endomorphisms of varieties and schemes........

Richard Hindman Bouldin, A generalization of the Weinstein-Aronszajn

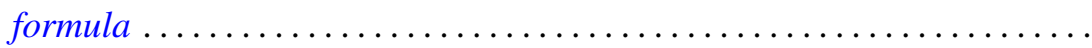

John Martin Chadam, The asymptotic behavior of the Klein-Gordon equation with external potential. II ...............................

Rina Hadass, On the zeros of the solutions of the differential equation

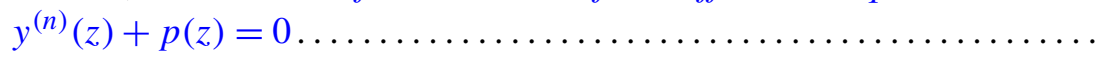

John Sollion Hsia, Integral equivalence of vectors over local modular lattices. II .............................................

Robert Hughes, Boundary behavior of random valued heat polynomial

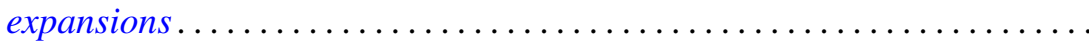

Surender Kumar Jain, Saad H. Mohamed and Surjeet Singh, Rings in which every right ideal is quasi-injective .........................

T. Kawata, On the inversion formula for the characteristic function .........

Erwin Kleinfeld, On right alternative rings without proper right ideals......

Robert Leroy Kruse and David Thomas Price, On the subring structure of

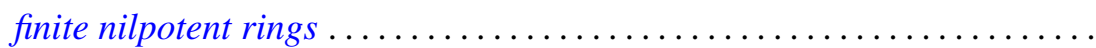

Marvin David Marcus and Stephen J. Pierce, Symmetric positive definite multilinear functionals with a given automorphism. .

William Schumacher Massey, Pontryagin squares in the Thom space of a bundle...

William Schumacher Massey, Proof of a conjecture of Whitney ...

John William Neuberger, Existence of a spectrum for nonlinear transformations

Stephen E. Newman, Measure algebras on idempotent semigroups ...

$\mathrm{K}$. Chandrasekhara Rao, Matrix transformations of some sequence spaces

Robert Bruce Schneider, Some theorems in Fourier analysis on symmetric

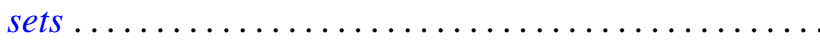

Ulrich F. K. Schoenwaelder, Centralizers of abelian, normal subgroups of hypercyclic groups...

Jerrold Norman Siegel, $G$-spaces, $H$-spaces and $W$-spaces

Robert Irving Soare, Cohesive sets and recursively enumerable Dedekind cuts...

Kwok-Wai Tam, Isometries of certain function spaces .... . .

Awadhesh Kumar Tiwary, Injective hulls of semi-simple modules over regular rings ....

Eldon Jon Vought, Concerning continua not separated by any nonaposyndetic subcontinuum .................... 